\title{
Systematic prediction of the biological functions of TAS2R10 using positive co-expression analysis
}

\author{
HONGLING ZHU ${ }^{1 *}$, LIANYONG LIU ${ }^{2 *}$, LI REN $^{1 *}$, JUNHUA MA $^{1 *}$, SHUANGGANG HU ${ }^{3}$, ZHAOHUI ZHU $^{1}$, \\ XUEMEI ZHAO ${ }^{1}, \mathrm{CHAO}^{\mathrm{SHI}}{ }^{1}$, XING WANG ${ }^{1}, \mathrm{CHAOBAO} \mathrm{ZHANG}^{4}$, MINGJUN GU $^{1}$ and XIANGQI LI ${ }^{1}$ \\ ${ }^{1}$ Department of Endocrinology, Shanghai Gongli Hospital, The Second Military Medical University, Shanghai 200135; \\ ${ }^{2}$ Department of Endocrinology, Punan Hospital of Pudong New District, Shanghai 200125; ${ }^{3}$ Shanghai Key Laboratory for \\ Assisted Reproduction and Reproductive Genetics, Center for Reproductive Medicine, Renji Hospital, School of Medicine, \\ Shanghai Jiao Tong University, Shanghai 200135; ${ }^{4}$ State Key Laboratory of Molecular Biology, CAS Center for \\ Excellence in Molecular Cell Science, Shanghai Institute of Biochemistry and Cell Biology, Chinese \\ Academy of Sciences, University of Chinese Academy of Sciences, Shanghai 200031, P.R. China
}

Received May 1, 2019; Accepted November 7, 2019

DOI: $10.3892 /$ etm.2019.8397

\begin{abstract}
Type 2 taste receptor 10 (TAS2R10), belonging to the TAS2R family of bitter receptors, is widely expressed in extra-oral tissues. However, its biological roles beyond bitterness sensing in the tongue have remained largely elusive. The present study aimed to perform a positive co-expression analysis using 60,000 Affymetrix expression arrays and 5,000 The Cancer Genome Atlas datasets to uncover such roles. Based on the functional enrichment analysis, it was indicated that in the Gene Ontology (GO) category biological process, TAS2R10 was mostly involved in 'cellular protein metabolic process', 'protein modification process', 'cellular protein modification process' and 'cellular component assembly'. In the GO category cellular component, the co-expressed genes were accumulated in 'Spt-Ada-Gcn5 acetyltransferase (SAGA)-type complex' and 'SAGA complex', and in the category molecular function, they were concentrated in 'hexosaminidase activity', 'cytoskeletal adaptor activity', 'cyclin binding' and ' $\beta$-N-acetylhexosaminidase activity'. Of note, it was indicated that TAS2R10 may be involved in 'ubiquitin-mediated proteolysis', which may provide a starting point to fully investigate the detailed functions of TAS2R10 in the future. TAS2R10 was also indicated to be associated with human diseases, i.e. 'Salmonella infection'. Overall, the
\end{abstract}

Correspondence to: Dr Mingjun Gu or Dr Xiangqi Li, Department of Endocrinology, Shanghai Gongli Hospital, The Second Military Medical University, 219 Miao-Pu Road, Shanghai 200135, P.R. China E-mail: mingjun_gu@163.com

E-mail: lixq@sibs.ac.cn

${ }^{*}$ Contributed equally

Key words: bitter receptor, type 2 taste receptor 10, function, disease, co-expression analysis present study was the first to perform a comprehensive bioinformatics analysis of the functions of TAS2R10 and provide insight regarding the notion that this gene may have crucial roles beyond bitterness sensing.

\section{Introduction}

Bitter perception has a critical role in host defense, as it functions as a warning signal against the ingestion of toxic substances (1). The recognition of bitter substances is mediated by a family of $\mathrm{G}$ protein-coupled receptors called type 2 taste receptors (TAS2Rs), which are prominently expressed in the tongue and include $>20$ members. However, recent investigations revealed that TAS2Rs are widely expressed in extra-oral tissues, including the gastric and intestinal mucosa, respiratory tract, bladder, pancreas, testes and central nervous system $(2,3)$, indicating TAS2Rs may perform numerous other functions other than perception of bitter taste.

Based on their agonist spectra, TAS2Rs may be grouped into broadly, narrowly, and intermediately tuned receptors (4); TAS2R10, TAS2R14 and TAS2R46 are broadly tuned receptors (5) and each recognizes about one-third of the bitter components tested so far (6). As such, these broadly tuned receptors may considerably contribute to the overall bitter tasting ability and it may be speculated that each of them may have numerous biological functions for different bitter substances that may exert various effects in the human body. Recent investigations on TAS2R10 confirmed this speculation. TAS2R10 induces the relaxation of smooth muscles of the ileum; this action may be mediated by nitric oxide and $\mathrm{BKCa}$ channels (7). TAS2R10 also dilates the airways by inhibiting airway smooth muscle calcium oscillations and calcium sensitivity (8). Furthermore, TAS2R10 was reported to mediate relaxation of vascular smooth muscles (9). Of note, TAS2R10 also has a tumor suppressor role in human neuroblastoma cells (10).

Based on the aforementioned studies, the present study hypothesized that TAS2R10 has multiple biological functions. 
To prove this hypothesis, a bioinformatics analysis of genes positively co-expressed with TAS2R10 was performed using 60,000 Affymetrix expression arrays and 5,000 TCGA datasets. The analysis indicated that TAS2R10 is mostly involved in biological activities, including 'positive regulation of biological process', 'cellular protein metabolic process', 'protein modification process, 'cellular protein modification process' and 'cellular component assembly'. The most prominent terms in the category cellular component were 'Spt-Ada-Gcn5 acetyltransferase (SAGA)-type complex' and 'SAGA complex'. The major terms in the category molecular function were 'hexosaminidase activity', 'cytoskeletal adaptor activity', 'cyclin binding' and ' $\beta-\mathrm{N}$-acetylhexosaminidase activity'. Of note, it was indicated that TAS2R10 may be involved in 'ubiquitin-mediated proteolysis', which may provide a key direction for investigating the functions of TAS2R10 in the future. TAS2R10 was also suggested to be linked to human diseases, i.e. 'Salmonella infection'. To the best of our knowledge, the present study was the first to comprehensively reveal the potential biological roles of TAS2R10 and provide crucial insights into the notion that this gene may have critical roles in addition to the perception of bitterness.

\section{Materials and methods}

Collection of expression data and pre-processing. The gene expression data were collected and pre-processed as described previously (11). The required data were retrieved from the European Bioinformatics Institute database (https://www. ebi.ac.uk/) and incorporated CEL files were pre-processed using the robust multichip average normalization method. The value 0.25 of the standard deviation level was used as the cutoff to guarantee for high-quality data and to capture significantly correlated transcriptome information. TAS2R10 was performed using 60,000 Affymetrix expression arrays and 5,000 TCGA datasets.

Biological pathway analysis. Gene Ontology (GO) function and Kyoto Encyclopedia of Genes and Genomes (KEGG) pathway analyses were performed (11). Analysis of biological pathways was performed for the genes that exhibited the highest correlation with TAS2R10 expression levels. The associated parameters used were identical to those of a previous study (11).

Statistical analysis. The statistical analysis was performed as described previously (11). Pearson correlation analysis was performed to assess the correlation between TAS2R10 and other genes and the P-values were unmodified. The q-value package in $\mathrm{R}$ was utilized to implement multiple testing corrections. Genes with q-values of $<0.05$ were considered as significant co-expressed genes of TAS2R10.

\section{Results}

TAS2R10 may be involved in a variety of biological functions according to $G O$ analysis. To reveal the potential functions of TAS2R10, a big data bioinformatics analysis, namely a positive co-expression analysis using 60,000 Affymetrix expression arrays and 5,000 TCGA data sets, was performed. As indicated in Fig. 1, GO enrichment analysis indicated that TAS2R10 may be involved in a myriad of functional terms in the category biological process, and the top five processes were 'positive regulation of biological process', 'cellular protein metabolic process', 'protein modification process', 'cellular protein modification process' and 'cellular component assembly'. The top 5 enriched functional terms in the category cellular component included 'cytosol', 'cytoskeletal part', 'chromosomal part', 'intracellular ribonucleoprotein complex' and 'ribonucleoprotein complex'. The top five terms in the category molecular functions were 'catalytic activity', 'enzyme binding', 'transcription factor activity', 'sequence-specific DNA binding', 'nucleic acid binding transcription factor activity' and 'zinc ion binding'.

To provide further information on the functions of TAS2R10, the top 20 items were revealed by searching the databases based on the GO analysis. Fig. 2 provides the top 20 biological process terms obtained using enrichment analysis. The top 20 biological processes included 'regulation of wound healing', 'spreading of epidermal cells', 'regulation of interleukin-12 secretion', 'regulation of gluconeogenesis by regulation of transcription from RNA polymerase II promoter', 'regulation of basement membrane assembly involved in embryonic body morphogenesis', 'protein retention in Golgi apparatus', 'positive regulation of peroxisome proliferator activated receptor signaling pathway', 'positive regulation of mitotic sister chromatid separation', 'positive regulation of mitotic metaphase/anaphase transition', 'positive regulation of hematopoietic stem cell proliferation', 'positive regulation of gluconeogenesis by positive regulation of transcription from RNA polymerase II promoter', 'positive regulation of extracellular matrix disassembly', 'positive regulation of brown fat cell differentiation', 'positive regulation of basement membrane assembly involved in embryonic body morphogenesis', 'negative regulation of hematopoietic progenitor cell differentiation', 'microRNA (miRNA) loading onto retinoid inducible serine carboxypeptidase involved in gene silencing by miRNA', 'microtubule anchoring at microtubule organizing center', 'histone H4-K20 trimethylation', 'coenzyme transport', 'cardiac pacemaker cell differentiation' and 'basement membrane assembly involved in embryonic body morphogenesis' (Fig. 2). The most prominent terms in the category cellular component were 'SAGA-type complex' and 'SAGA complex' (Fig. 3). The molecular functions covered 'hexosaminidase activity', 'cytoskeletal adaptor activity', 'cyclin binding' and ' $\beta$-N-acetylhexosaminidase activity' (Fig. 4). Furthermore, in order to confirm the results of the bioinformatics analysis, the expression of TAS2R10 was examined in different human cell lines, HeLa, TPC1, CAPAN-2, HEK293, HEPG2, A549, Caco-2, MCF7 and RT4 (Bena Culture Collection) by reverse transcription-quantitative (RT-q)PCR and western blot analysis (Fig. S1). It was indicated that TAS2R10 was mainly expressed in the cell lines HeLa, TPC1 and CAPAN-2 at different levels, suggesting that TAS2R10 may have different functions in different tissue types. It may perform biological functions in the tissues of uterus, thyroid and pancreas, and exhibit no functions in other tissues, kidney, liver, lung, colon, breast, and bladder.

TAS2R10 may take part in various signaling pathways according to KEGG analysis. To further identify the pathways 


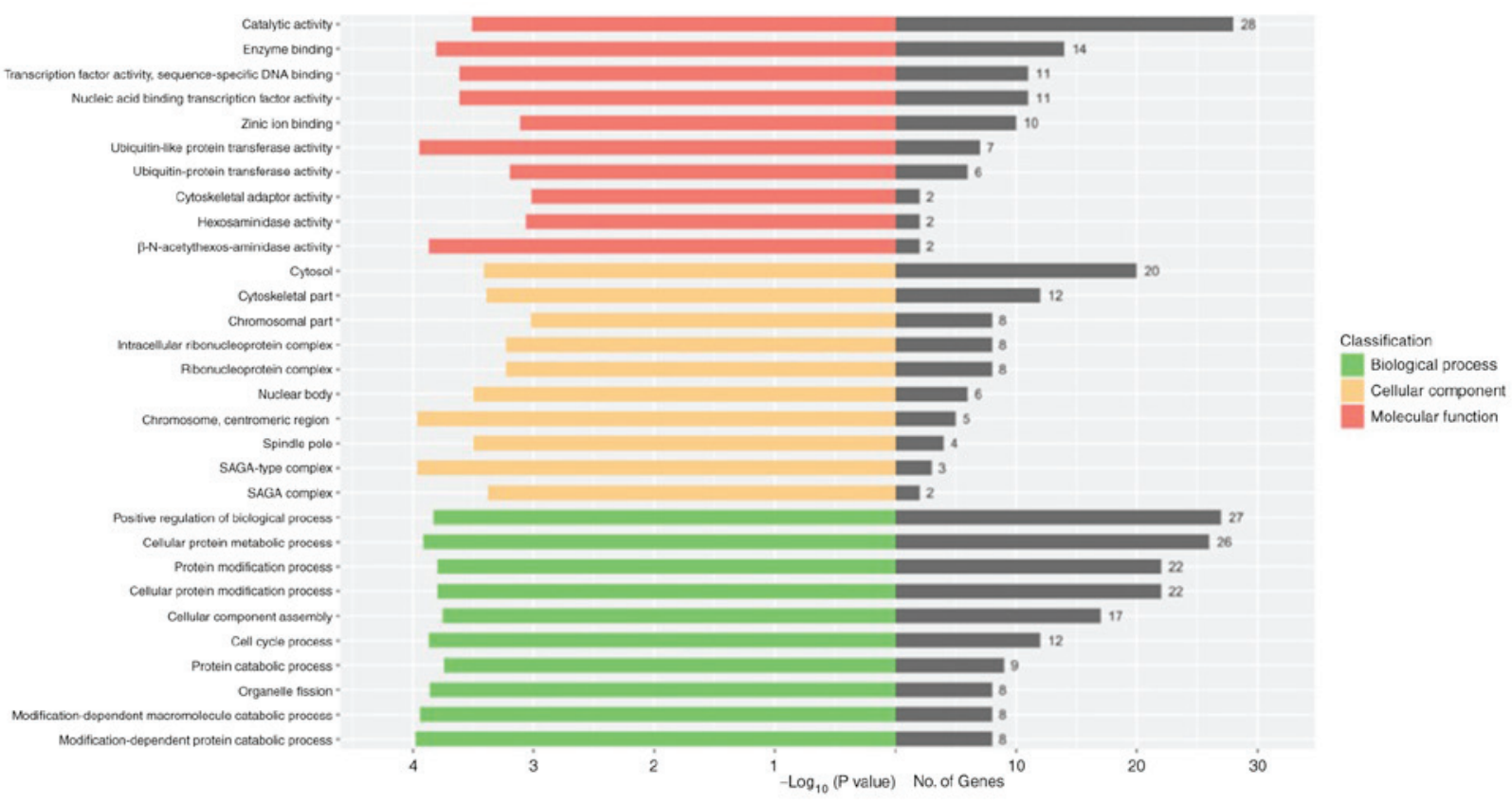

Figure 1. Functional roles of TAS2R10 according to Gene Ontology analysis indicated by classification. Biological functions were identified by enrichment analysis of genes positively co-expressed with TAS2R10 in 60,000 Affymetrix expression arrays and 5,000 datasets from the Cancer Genome Atlas. TAS2R10, type 2 taste receptor 10 .

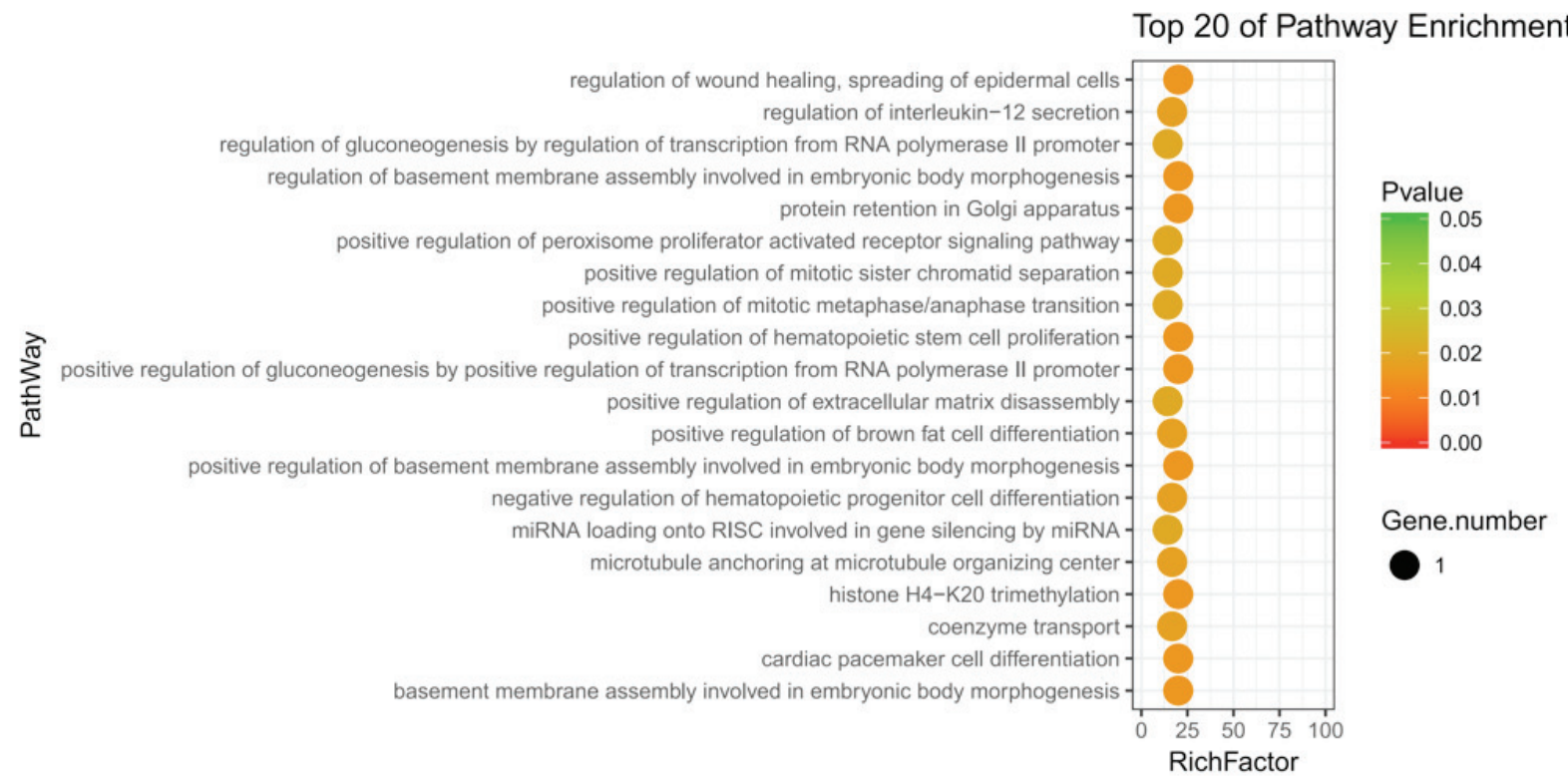

Figure 2. Top 20 functional terms in the category biological process of TAS2R10 according to Gene Ontology analysis. Enrichment analysis of genes co-expressed with TAS2R10 was performed using 60,000 Affymetrix expression arrays and 5,000 The Cancer Genome Atlas datasets. TAS2R10, type 2 taste receptor 10.

involving TAS2R10, a KEGG analysis was performed based on the genes positively co-expressed with TASR10 (Fig. 5). In the item 'organismal systems', TAS2R10 was positively associated with 'retrograde endocannabinoid signaling' and 'progesterone-mediated oocyte maturation'. In the item 'genetic information processing, TAS2R10 was positively linked to 'ubiquitin-mediated proteolysis'. In addition, TAS2R10 was indicated to be involved in numerous metabolic activities, including 'glycosphingolipid biosynthesis-ganglio series', 'other glycan degradation', 'glycosaminoglycan degradation' and 'glycosphingolipid biosynthesis-globo series'. Of note, TAS2R10 was indicated to be involved in certain pathogenic processes/human diseases, specifically 'Salmonella infection'. These functional pathways are not only associated with the tongue but also are involved in extra-oral functions.

To provide further information on the signaling pathways of TAS2R10, an enrichment analysis was used to determine the top 20 pathways (Fig. 6). The top five pathways are 'ubiquitin mediated proteolysis', 'Salmonella infection', 'retrograde endocannabinoid signaling', 'progesterone-mediated oocyte 


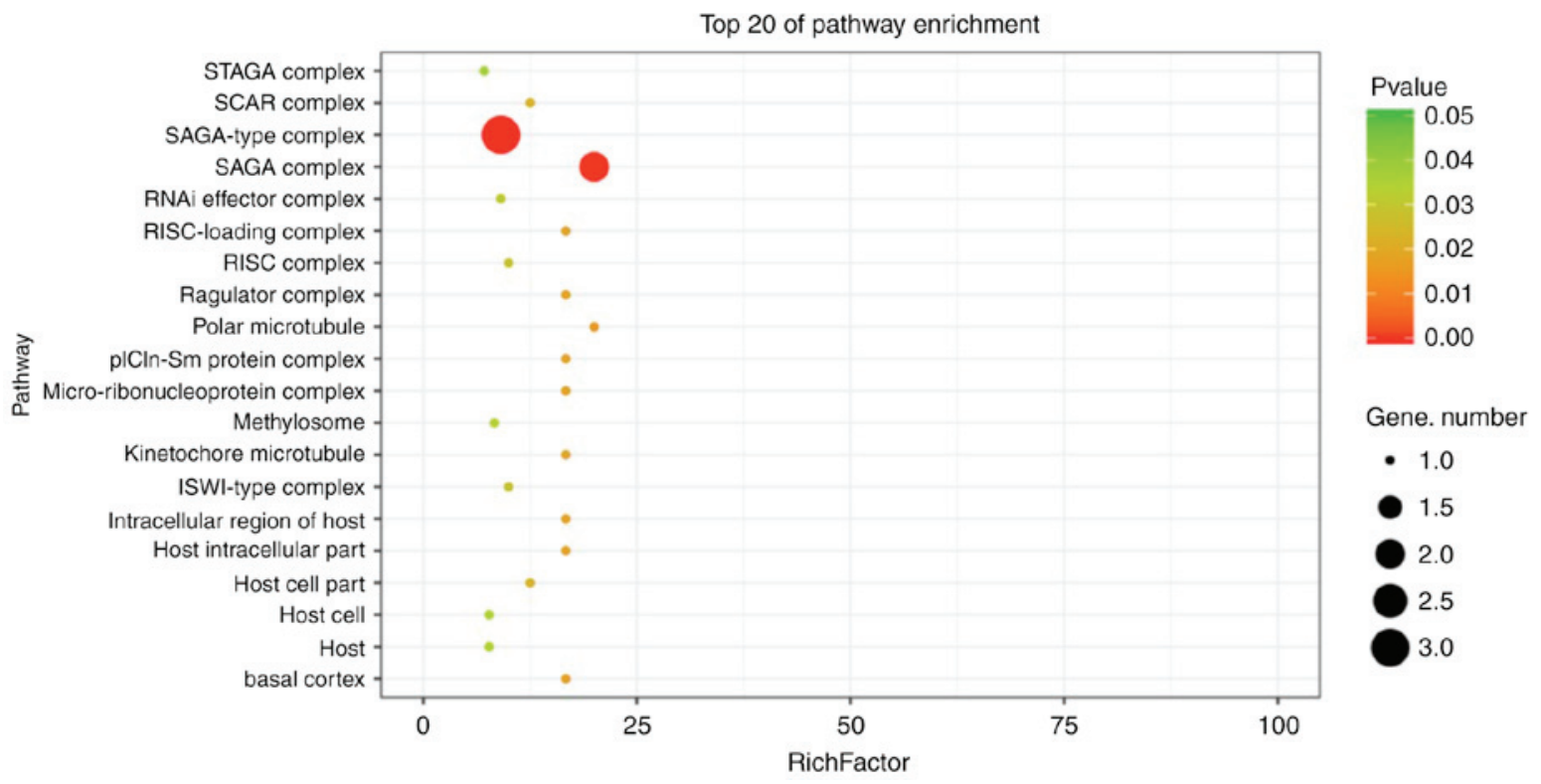

Figure 3. Top 20 functional terms in the category cellular component of TAS2R10 based on Gene Ontology analysis. Enrichment analysis of genes co-expressed with TAS2R10 was performed using 60,000 Affymetrix expression arrays and 5,000 The Cancer Genome Atlas datasets. The most distinct terms were 'SAGA-type complex' and 'SAGA complex'. TAS2R10, type 2 taste receptor 10; SAGA, Spt-Ada-Gen5 acetyltransferase; RISC, retinoid inducible serine carboxypeptidase; RNAi, RNA interference.

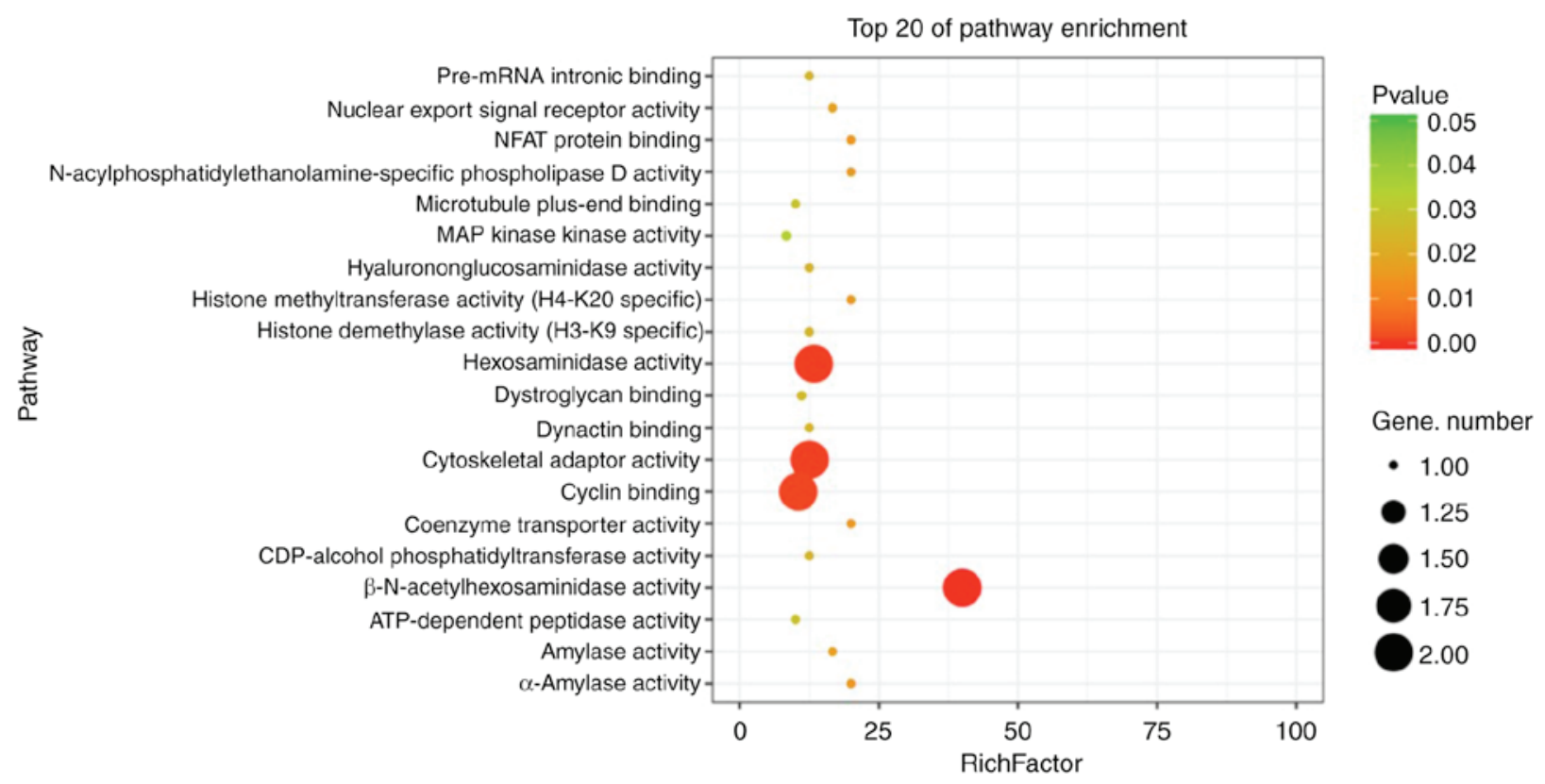

Figure 4. Top 20 functional terms in the category molecular function of TAS2R10 based on Gene Ontology analysis. Enrichment analysis of genes co-expressed with TAS2R10 was performed using 60,000 Affymetrix expression arrays and 5,000 The Cancer Genome Atlas datasets. The key terms were 'hexosaminidase activity', 'cytoskeletal adaptor activity', 'cyclin binding' and ' $\beta$-N-acetylhexosaminidase activity'. TAS2R10, type 2 taste receptor 10.

maturation' and 'glycosphingolipid biosynthesis-ganglio series'. Among these pathways, 'ubiquitin mediated proteolysis' ranked first, followed by 'glycosphingolipid biosynthesis-ganglio series'. For ubiquitin-mediated pathway, the potential targets for TAS2R10 include anaphase promoting complex subunit 5 (ANAPC5) and ubiquitin protein ligase E3B were predicted. Furthermore, in order to confirm the results of the bioinformatics analysis, the association of TAS2R10 and ANAPC5 in human thyroid was examined by using RT-qPCR (Fig. S2). ANAPC5 is required for the proper ubiquitination function (12). It was indicated that the expression levels of TAS2R10 were significantly associated with those of ANAPC5, suggesting TAS2R10 may be involved in the ubiquitin pathway.

\section{Discussion}

Bitterness sensing is mediated by the TAS2R family occurring in the tongue (1). The wide expression of TAS2Rs in tissues other than the tongue has been reported in recent 


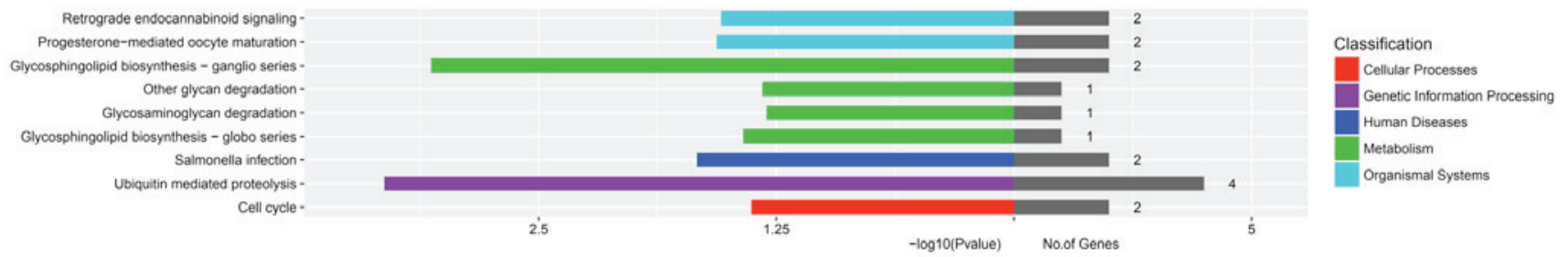

Figure 5. Signaling pathways for TAS2R10 determined by Kyoto Encyclopedia of Genes and Genomes analysis indicated by classification. Enrichment analysis of genes co-expressed with TAS2R10 was performed using 60,000 Affymetrix expression arrays and 5,000 The Cancer Genome Atlas datasets. TAS2R10 was indicated to be involved in a variety of signaling pathways. TAS2R10, type 2 taste receptor 10.

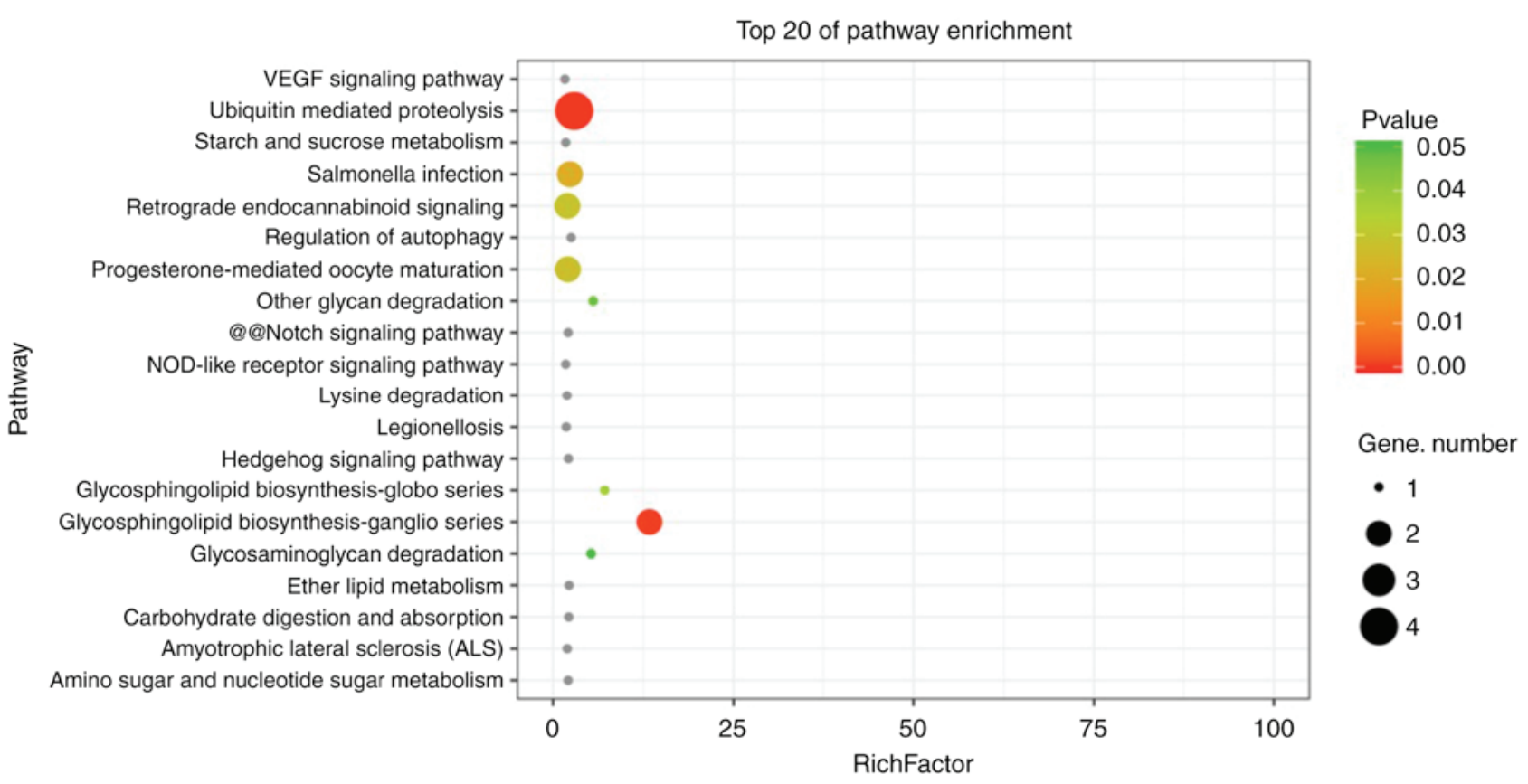

Figure 6. Top 20 signaling pathways for TAS2R10 determined by Kyoto Encyclopedia of Genes and Genomes analysis. Enrichment analysis of genes co-expressed with TAS2R10 was performed using 60,000 Affymetrix expression arrays and 5,000 The Cancer Genome Atlas datasets. The top five pathways included 'ubiquitin mediated proteolysis', 'Salmonella infection', 'retrograde endocannabinoid signaling', 'progesterone-mediated oocyte maturation' and 'glycosphingolipid biosynthesis-ganglio series'. TAS2R10, type 2 taste receptor 10.

years $(2,3)$. Thus, TAS2Rs were postulated to have extra-oral biological roles. However, at present, functional investigations are limited and scattered. In the present study, a comprehensive investigation of the potential functions of TAS2R10 was performed by positive co-expression analysis using 60,000 Affymetrix expression arrays and 5,000 TCGA datasets. Various noteworthy results were obtained. First, TAS2R10 may be involved in various biological activities beyond the perception of bitterness with a focus on protein modification and metabolic processes. The present experimental results confirmed that TAS2R10 was mainly expressed in the cell lines HeLa, TPC1 and CAPAN-2, suggesting that TAS2R10 may be involved in different biological activities in different tissues. Furthermore, major GO terms in the category cellular component were 'SAGA-type complex' and 'SAGA complex'. In addition, 'ubiquitin mediated proteolysis' is a typical pathway involving TAS2R10. Finally, TAS2R10 may be involved in 'Salmonella infection'. With regard to the ubiquitin pathway, the potential targets for TAS2R10 include ANAPC5 and ubiquitin protein ligase E3B based on the present bioinformatics analysis. ANAPC5 is a ubiquitin ligase that controls cell cycle progression through ubiquitination (12). In the present study, the association of TAS2R10 and ANAPC5 in human thyroid was confirmed by RT-qPCR. These results indicate that TAS2R10 may be involved in the ubiquitin pathway through ANAPC5.

TAS2R10 is known to be a broadly tuned bitter receptor (5), and it was able to recognize approximately one-third of the bitter components tested thus far (6). It may be speculated that TAS2R10 is able to perform numerous biological functions. Recent studies have indicated other functions of TAS2R10 than bitterness-sensing (10). However, only two functions have been reported. One function of TAS2R10 is that it is able to induce the relaxation of smooth muscles of the ileum (7), airway (8) and blood vessels (9). In the present study, it was observed that TAS2R10 features cytoskeletal adaptor activity and is also associated with hexosaminidase activity. These activities are linked to the role of smooth muscles. The second function reported for TAS2R 10 was its tumor-suppressor role (10). In the present study, TAS2R10 was indicated to be involved in 'cellular processes of cell cycle' and may thus exert a regulatory function in cancer. 
In conclusion, through positive co-expression analysis using 60,000 Affymetrix expression arrays and 5,000 TCGA datasets, the present study not only confirmed the previously reported functions but also revealed novel roles of TAS2R10. It was observed that TAS2R10 is associated with 'ubiquitin mediated proteolysis' and 'Salmonella infection', which may serve as a reference for detailed investigation of the functions of TAS2R10 in the future. To the best of our knowledge, the present study was is the first to comprehensively investigate the biological functions of TAS2R10, providing crucial insight into the concept that this gene may have critical roles other than bitterness sensing.

\section{Acknowledgements}

Not applicable.

\section{Funding}

The present study was supported and the publication costs were covered in part by grants from Specialty Feature Construction Project of Pudong Health and Family Planning Commission of Shanghai (grant no. PWZzb2017-22), Key Discipline Construction Project of Pudong Health and Family Planning Commission of Shanghai (grant no. PWZxk2017-07), Key Specialty Construction Project of Pudong Health and Family Planning Commission of Shanghai (grant no. PWZzk2017-29), Key Disciplines Group Construction Project of Pudong Health and Family Planning Commission of Shanghai (grant no. PWZxq2017-17), Pudong New Area Science and Technology Commission (grant nos. PKJ2019-Y21, PKJ2016-Y03 and PKJ2018-Y08), and Shanghai Health and Family Planning Commission (grant no. 201640177), Outstanding Leaders Training Program of Pudong Health Bureau of Shanghai (grant no. PWR12018-02).

\section{Availability of data and materials}

The datasets generated and/or analyzed during the present study are available from the corresponding author on reasonable request.

\section{Authors' contributions}

HZ, MG and XL conceived and designed the study; SH and $\mathrm{ZZ}$ collected the data; $\mathrm{XZ}, \mathrm{CS}, \mathrm{XW}$ and $\mathrm{CZ}$ performed the analysis; LR, LL and JM wrote the manuscript and performed the experiments All of the authors have read and approved the final manuscript.

\section{Ethics approval and consent to participate}

The present study was approved by the Ethics Committee of Punan Hospital of Pudong New District (Shanghai, China). All of the patients provided written informed consent to participate in the present study.

\section{Patient consent for publication}

Not applicable.

\section{Competing interests}

The authors declare that they have no competing interests.

\section{References}

1. Li D and Zhang J: Diet shapes the evolution of the vertebrate bitter taste receptor gene repertoire. Mol Biol Evol 31: 303-309, 2014

2. Kook JH, Kim HK, Kim HJ, Kim KW, Kim TH, Kang KR, Oh DJ and Lee SH: Increased expression of bitter taste receptors in human allergic nasal mucosa and their contribution to the shrinkage of human nasal mucosa. Clin Exp Allergy 46: 584-601, 2016.

3. Colombo M, Trevisi P, Gandolfi G and Bosi P: Assessment of the presence of chemosensing receptors based on bitter and fat taste in the gastrointestinal tract of young pig. J Anim Sci 90 (Suppl 4): S128-S130, 2012.

4. Behrens M and Meyerhof W: Bitter taste receptor research comes of age: From characterization to modulation of TAS2Rs. Semin Cell Dev Biol 24: 215-221, 2013.

5. Nowak S, Di Pizio A, Levit A, Niv MY, Meyerhof W and Behrens M: Reengineering the ligand sensitivity of the broadly tuned human bitter taste receptor TAS2R14. Biochim Biophys Acta Gen Subj 1862: 2162-2173, 2018.

6. Meyerhof W, Batram C, Kuhn C, Brockhoff A, Chudoba E, Bufe B, Appendino G and Behrens M: The molecular receptive ranges of human TAS2R bitter taste receptors. Chem Senses 35: 157-170, 2010.

7. Jing F, Liu M, Yang N, Liu Y, Li X and Li J: Relaxant effect of chloroquine in rat ileum: Possible involvement of nitric oxide and BKCa. J Pharm Pharmacol 65: 847-854, 2013.

8. Tan X and Sanderson MJ: Bitter tasting compounds dilate airways by inhibiting airway smooth muscle calcium oscillations and calcium sensitivity. Br J Pharmacol 171: 646-662, 2014.

9. Manson ML, Safholm J, Al-Ameri M, Bergman P, Orre AC, Swärd K, James A, Dahlèn SE and Adner M: Bitter taste receptor agonists mediate relaxation of human and rodent vascular smooth muscle. Eur J Pharmacol 740: 302-311, 2014.

10. Seo Y, Kim YS, Lee KE, Park TH and Kim Y: Anti-cancer stemness and anti-invasive activity of bitter taste receptors, TAS2R8 and TAS2R10, in human neuroblastoma cells. PLoS One 12: e0176851, 2017

11. Chen M, Wang J, Luo Y, Huang K, Shi X, Liu Y, Li J, Lai Z, Xue $\mathrm{S}$, Gao $\mathrm{H}$, et al: Identify Down syndrome transcriptome associations using integrative analysis of microarray database and correlation-interaction network. Hum Genomics 12: 2, 2018.

12. Schreiber A, Stengel F, Zhang Z, Enchev RI, Kong EH, Morris EP, Robinson CV, da Fonseca PC and Barford D: Structural basis for the subunit assembly of the anaphase-promoting complex. Nature 470: 227-232, 2011.

This work is licensed under a Creative Commons Attribution-NonCommercial-NoDerivatives 4.0 International (CC BY-NC-ND 4.0) License. 